\title{
Modified high power impulse magnetron sputtering process for increased deposition rate of titanium
}

\author{
Paul Michael Barker ${ }^{a}$ \\ Laboratory for Nanoscale Materials Science, Empa, Dübendorf, Switzerland \\ Erik Lewin \\ Laboratory for Nanoscale Materials Science, Empa, Dübendorf, Switzerland and Department of Chemistry, \\ Ångström Laboratory, Uppsala University, Sweden \\ Jörg Patscheider \\ Laboratory for Nanoscale Materials Science, Empa, Dübendorf, Switzerland
}

(Received 2 July 2013; accepted 13 August 2013; published 29 August 2013)

\begin{abstract}
A modified version of high power impulse magnetron sputtering (HiPIMS) has been used to deposit titanium films at higher deposition rates than for conventional HiPIMS while maintaining similar pulse voltages and peak currents. In the present study, additional control parameters are explored through the chopping of the HiPIMS pulse into a pulse sequence. Experiments show that the use of sequences allows for an increase of the deposition rate of more than $45 \%$ compared to conventional HiPIMS. The increase in deposition rate is ascribed to a combination of reduced gas rarefaction effects, prevention of sustained self-sputtering, and a relaxation of ion trapping. (C) 2013 American Vacuum Society. [http://dx.doi.org/10.1116/1.4819296]
\end{abstract}

\section{INTRODUCTION}

High power impulse magnetron sputtering (HiPIMS) has become a well known tool for PVD since gaining popularity through the seminal work by Kouznetsov et al. ${ }^{1}$ in 1999. Being essentially a variant of direct current magnetron sputtering (DCMS), HiPIMS may be carried out in a same or similar coating setup as DCMS. The voltage is applied in high power pulses with low duty cycles in the range of $0.5-5 \%$ and peak powers that can reach the megawatt range. During the short (20-200 $\mu$ s) high power peaks, a high level of ionization can be reached. The increased ion-to-atom ratio attainable in HiPIMS has the effect of a "more efficient and accurate control of bombarding conditions" allowing tailoring of the coatings properties produced by this method. Films produced by HiPIMS are reported to have various improved characteristics when compared to DCMS, ${ }^{3,4}$ as well as processing benefits such as reduced hysteresis in reactive mode ${ }^{5}$ and low thermal flux delivered to the substrate. ${ }^{6}$ HiPIMS technology is explained in more detail in Refs. 2, 7-9.

Despite improved control of the structure and properties of the films produced by HiPIMS, industrial acceptance of the process has been slow. A part of this reluctance is due to reports of low deposition rates for most materials, when comparing HiPIMS to DCMS, a summary of which is available in Ref. 4. There is a large number of possible reasons for this reduced deposition rate, which are discussed in Refs. 2, 7-9. Some methods have been put forward to tackle this issue such as the use of medium frequency superimposed HiPIMS (Ref. 10) or modulated pulsed power (MPP) magnetron sputtering. ${ }^{11}$ Other, more innovative but complex approaches have also been employed to increase HiPIMS

${ }^{\text {a)} E l e c t r o n i c ~ m a i l: ~ P a u l . B a r k e r @ e m p a . c h ~}$ deposition rates, such as the addition of electron cyclotron wave resonance to the HiPIMS system, ${ }^{12}$ but as yet no single approach has become established. This letter reports a novel approach to HiPIMS which, with the relevant power supply, is simple to implement while resulting in a significantly increased deposition rate for titanium by HiPIMS.

\section{EXPERIMENT}

For the experiments, a Melec SIPP2000 HiPIMS pulsing unit has been used, powered by an Advanced Energy Pinnacle DC supply. This pulser allows the decomposition of a single HiPIMS pulse into several individual pulses to produce pulse sequences, referred to as chopped-HiPIMS (c-HiPIMS). Figure 1 shows a comparison of a HiPIMS pulse (lower) and two different c-HiPIMS pulses. The effect of adjusting the delay between the micropulses, the micropulse off-time, has been investigated with respect to the effect on deposition rate.

It should be noted that, while a standard HiPIMS waveform is decomposed, the approach is different to MPP another modified HiPIMS process described in Ref. 11. The main differences between c-HiPIMS and MPP are the number of decompositions of the pulse-on (which are fewer in number for c-HiPIMS), lack of an ignition stage as used in MPP, and the repetition frequency and total pulse-on times, values of which fall within the average HiPIMS range for c-HiPIMS.

The c-HiPIMS pulse is denoted as follows: in $\mathrm{yy}(\mathrm{xx}) * \mathrm{n}$ yy stands for the $t_{\mu o n}$, ( $\left.x x\right)$ represents the $t_{\mu o f f}$ between micropulses, and $*_{n}$ represents the number of micropulses within the macropulse. In this manner, a c-HiPIMS pulse containing four micropulses of $25 \mu \mathrm{s}$ with a $\mathrm{t}_{\mu \mathrm{off}}$ of $50 \mu \mathrm{s}$ are described as $25(50)^{*} 4$, with a total $\mathrm{t}_{\text {on }}$ of $100 \mu \mathrm{s}$. Two cHiPIMS pulse sequences have been investigated: $25(\mathrm{xx}) * 4$ and $50(\mathrm{xx}) * 2$ as well as a HiPIMS reference, $100 * 1$. The $\mathrm{t}_{\mu \text { off }}$ has been varied from 10 to $300 \mu$ s for the experimental series reported. In all three cases the same $t_{\text {on }}, 100 \mu \mathrm{s}$, 


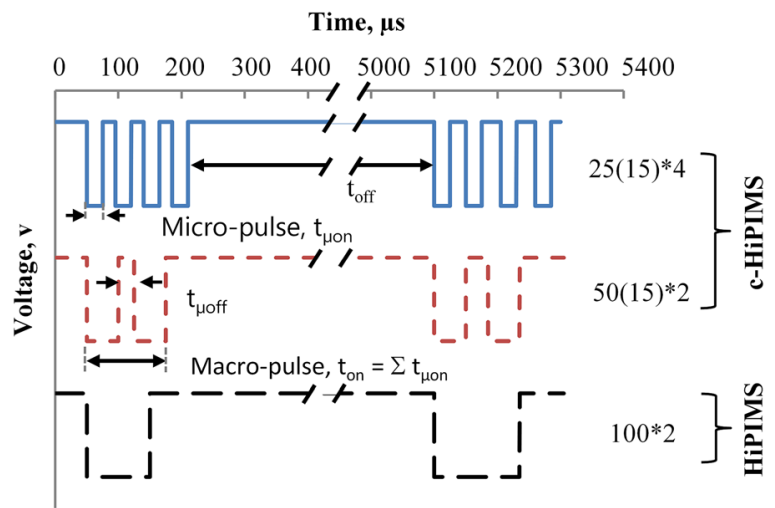

FIG. 1. (Color online) Graph showing c-HiPIMS pulses consisting of micropulses (typical for the present study) and a HiPIMS pulse for comparison.

frequency, $200 \mathrm{~Hz}$, and time-averaged power, $0.75 \mathrm{~kW}$, was used. The $t_{\text {off }}$ has been adjusted in order to maintain a constant repetition frequency. As the $\mathrm{t}_{\text {on }}$ time is maintained, at $100 \mu$ s, the duty cycle is also kept constant.

The system used here contains four magnetron sources $(88 \times 300 \mathrm{~mm})$ mounted at an angle of $90^{\circ}$ to each other at the periphery of a cylindrical chamber $(400 \mathrm{~mm}$ in diameter), arranged in closed field configuration. A single Ti cathode (purity 99.9\%) was powered via the Melec HiPIMS pulser. Discharge voltage and currents were monitored but are not reported here. Prior to deposition, the chamber has been evacuated to a pressure of $<1 \cdot 10^{-3} \mathrm{~Pa}$ then backfilled with argon to a working pressure of $0.65 \mathrm{~Pa}$. A quartz crystal microbalance (QCM), mounted $100 \mathrm{~mm}$ from the cathode, was used to measure deposition rates. Thin films were grown on floating substrates, and it was seen that deposition rate trends matched the QCM measurements. X-ray diffraction (XRD) investigations showed that the films grow in a strongly preferred (111) orientation; XPS depth profiles showed that only titanium and oxygen are present in the films, the latter in concentrations below 1 at. \%. In-depth discussion of these films is beyond the scope of this letter, although scanning electron microscopy and XRD measurements showed that all films were dense and had no significant differences among themselves.

\section{RESULTS AND DISCUSSION}

Figure 2 shows the voltage and current waveforms for the four different run conditions; standard HiPIMS (a), c-HiPIMS with four $t_{\mu \circ n}$ repetitions (b), and c-HiPIMS with two $t_{\mu o n}$ repetitions but for varied $t_{\mu \text { off }}$ times (c) and (d). All the waveforms in Fig. 2 have the same $t_{\mathrm{on}}, 100 \mu$ s, repetition frequency, $200 \mathrm{~Hz}$, and time averaged power, $\mathrm{P}_{\mathrm{tav}}, 0.75 \mathrm{~kW}$. It can be seen from the voltage curves that the use of c-HiPIMS and HiPIMS has no significant effect on the level of average pulse voltage used to achieve $0.7 \mathrm{~kW}$, with similar values used in all cases. In the same way, the current waveforms show similar values for both peak and average currents for c-HiPIMS and HiPIMS. However, in Fig. 2(b), it can be seen that during the first micropulse the peak current reached is lower than the subsequent pulses. The same
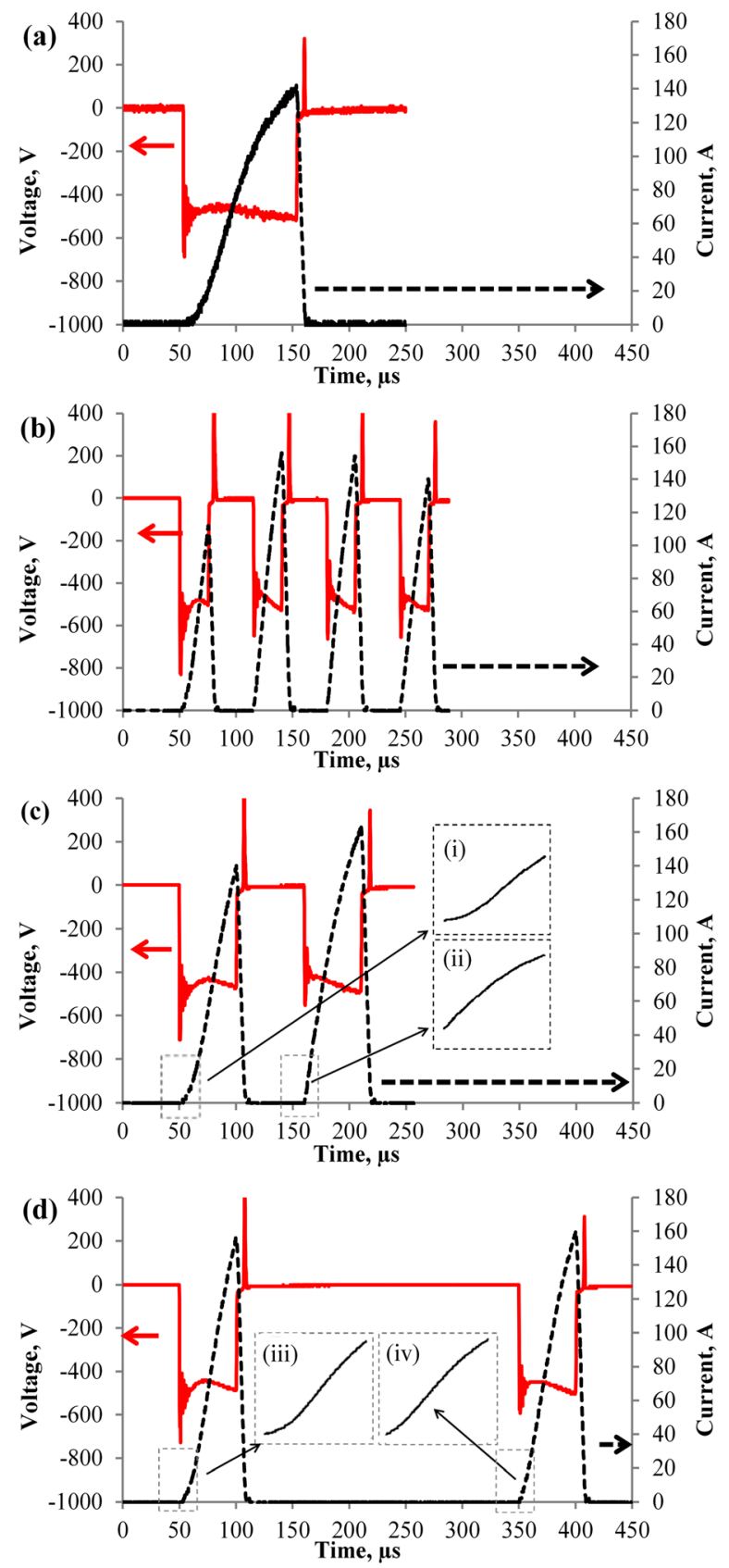

FIG. 2. (Color online) Voltage (top) and current (bottom) traces recorded using an oscilloscope, showing the experimental waveforms for (a) $200 * 1$, (b) $25(40) * 4$, (c) $50(40) * 2$, and (d) $50(250) * 2$. Insets [c(i and ii)] and [d(iii and iv)] are expanded images of the rising current curves for the areas highlighted in the main figure where the first points of the expanded curves indicate the start of the voltage pulse.

can be seen in Fig. 2(c), where insets (i) demonstrate that there is a delay in the evolution of the current curves once the voltage is applied to the cathode. This is due to a delay of the current onset during the first pulse, described by Anders as the time for an ion current to be established within the system. ${ }^{13}$ It seems that after the first micropulse, inset (ii), a sufficient number of ions remains within the near cathode region. For the subsequent pulse, there are already charge carriers available for generating electrons and ion avalanches, resulting in little delay in the current evolution, as shown in Fig. 2(c-ii). Given the delay in current onset, it 
is no surprise that the peak current achieved for the first micropulse is lower than for consequent micropulses. While the current evolution in Fig. 2(d-iv) may experience a slight delay compared to (ii), the delay is smaller than for the first micropulse, inset (iii). Despite the relatively long $t_{\mu \text { off }}$ time of $250 \mu \mathrm{s}$, this shows that ions remain in the near-cathode region for a significant time after the voltage to the cathode is reduced to zero.

Figure 2(b) also shows that the final $\mathrm{t}_{\mu \text { on }}$ pulse has a lower peak current than the second and third. This is likely due to a reduction of the pressure of the working gas, argon, in the near target region. It is depleted in this region due to accumulated heating, an effect that will be discussed further below. Similar trends in current evolution and magnitudes are observed for various $\mathrm{t}_{\mu \text { off }}$ times for the $25(\mathrm{xx}) * 4$ pulse as well as $50(\mathrm{xx}) * 2$.

Figure 3 shows the effect of increasing $\mathrm{t}_{\mu \mathrm{off}}$ on the deposition rate, when using c-HiPIMS. The deposition rate for the two c-HiPIMS pulses increases as $t_{\mu \text { off }}$ increases until the deposition rate levels off at $\mathrm{t}_{\mu \mathrm{off}} \sim 250 \mu \mathrm{s}$. For the $50(\mathrm{xx}) * 2$ c-HiPIMS pulse (squares), the deposition rate rises from $2.8 \AA / \mathrm{s}$ at $\mathrm{t}_{\mu \text { off }}=10 \mu$ s to $3.2 \AA / \mathrm{s}$ at $\mathrm{t}_{\mu \text { off }}=250 \mu \mathrm{s}$, which corresponds to an increase of $15 \%$, and an increase of $23 \%$ compared to HiPIMS. For $25(\mathrm{xx}) * 4$ (triangles) the increase is even more pronounced, with an enhancement of $34 \%$ as the $\mathrm{t}_{\mu \mathrm{off}}$ time increases from 10 to $300 \mu \mathrm{s}$ and a maximum increase of $47 \%$ compared to HiPIMS. Nevertheless, the maximum deposition rate for c-HiPIMS is only $75 \%$ that of DCMS.

It is worth pointing out at this stage that $25(\mathrm{xx}) * 2$ at $200 \mathrm{~Hz}$ should not be compared to $25^{*} 1$ at higher frequencies. There are significant difference in the voltage and current values due to longer $\mathrm{t}_{\text {off }}$ times for c-HiPIMS $[25 * 1$ at $800 \mathrm{~Hz}$ has a $\mathrm{t}_{\text {off }}$ of $1200 \mu \mathrm{s}$ compared to $4650 \mu$ s for $50(250) * 2$ at $200 \mathrm{~Hz}$ and $4150 \mu$ s for $25(250)^{*} 4$ also at $200 \mathrm{~Hz}$. Further, the deposition rate for $25^{*} 1$ at $800 \mathrm{~Hz}$ was significantly lower (around $40 \%$ lower) than $25(250) * 4$ at $200 \mathrm{~Hz}$, but the discussion of these results are beyond the scope of this letter.

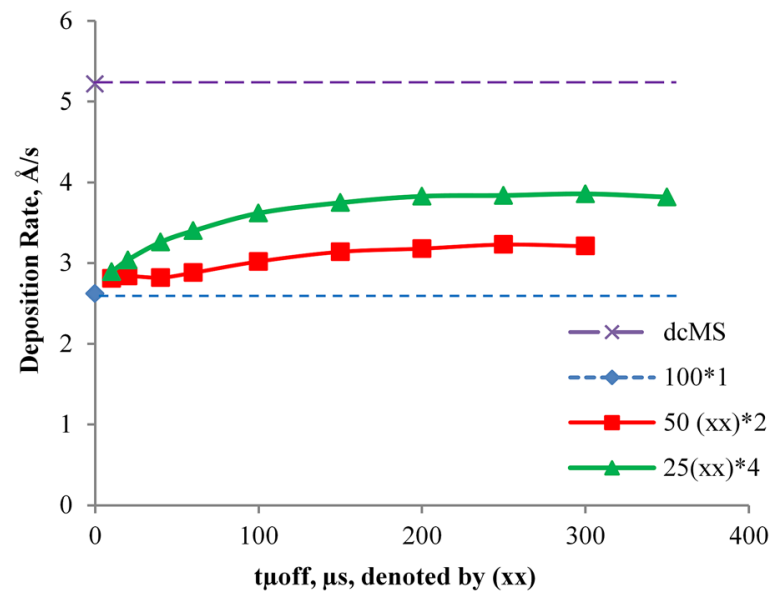

FIG. 3. (Color online) Graph showing the effect of $\mathrm{t}_{\mu \mathrm{off}}$ on deposition rate, measured using a QCM, for c-HiPIMS, HiPIMS, and DCMS. Note that the total time-on is the same for all points on the graph (except for the DCMS case).
Increased deposition rates were reported by Konstantinidis et al., ${ }^{14}$ when the length of the total on-time for a given frequency was decreased. The finding was explained by increased self-sputtering at longer pulse lengths. Simulations by Kadlec ${ }^{15}$ showed that at $\mathrm{t}_{\mathrm{on}}<50 \mu$ s there are significantly higher gas densities directly in front of the target. Longer pulses transfer more heat to the working gas, Ar. Higher gas temperatures result in higher levels of expansion (rarefaction) and therefore more depletion of $\mathrm{Ar}$ in the near-cathode region for longer pulses. ${ }^{16}$ Anders et al. ${ }^{13}$ showed that at long enough $t_{\text {on }}$ (as a single pulse), due to rarefaction the sputtering mechanism is dominated by sustained self-sputtering, which has a lower effective deposition rate than sputtering by argon bombardment for many materials. Lundin et al. ${ }^{17}$ suggest that during the pulse-off time a refill of the working gas takes place. This effect was explored further by Kubart et al. ${ }^{18}$ who showed that when higher frequencies are used with shorter times between the pulses, the first HiPIMS current pulse was of higher magnitude than the following pulse. This effect was diminished at lower frequency, which can be understood as a result of a more complete gas refill in the area directly in front of the target with longer times between pulses, resulting in higher peak currents.

According to Ref. 13, the 25 and $50 \mu$ s micropulses with c-HiPIMS avoid the onset of significant self-sputtering, compared to the $100 \mu$ s HiPIMS pulse. It may be assessed, however, that keeping $t_{\mu o n}$ short results in reduced rarefaction, and thus sufficient argon remains in the near cathode region to avoid the onset of significant self-sputtering. Since argon sputtering has a higher sputter yield than self-sputtering, the deposition rate will be higher than when using a $100 \mu$ s pulse, as seen in Fig. 3. While rarefaction may also be mitigated through a reduction of the time-averaged power supplied to a single HiPIMS pulse, the reduced time averaged power itself leads to reduced deposition rates for the same pulse shapes.

A further beneficial effect of using c-HiPIMS is that an opportunity for gas refilling in the near cathode area is introduced during the $t_{\mu \text { off }}$ times. The longer the $t_{\mu \text { off }}$, the more time is available for gas refill and thus an increase in the deposition rate is expected, as is seen in Fig. 3. At around $\mathrm{t}_{\mu \mathrm{off}}=250 \mu \mathrm{s}$, the deposition rate begins to level off. This suggests a counter-effect preventing the deposition rate from increasing further, as shown in Ref. 18, since a total gas refill cannot have occurred on this time scale.

Consideration may also be given to the trapping of the ionized species, recognized in HiPIMS as a contributing factor to reduced deposition rate. ${ }^{19}$ This effect occurs when the target voltage is turned on, as the strong electrical fields in the cathode sheath act as a trap confining the ionized sputtered flux and preventing ion transport to the substrate. The $t_{\mu \text { off }}$ between micropulses provides opportunities for the ion flux to "escape" this near cathode trap and reach the substrate. The more micro-pulses are used, the more $t_{\mu o f f}$ opportunities are presented. This further explains differences between the two c-HPIMS conditions displayed in Fig. 3 . Further, it can also explain the saturation of the deposition rate, if a maximum amount of the generated ion flux has been able to travel to the substrate within $\mathrm{t}_{\text {off }} \sim 250 \mu \mathrm{s}$. 
In this manner, it can be expected that when using $25(\mathrm{xx}) * 4$, there is less rarefaction during the $\mathrm{t}_{\mu \mathrm{on}}$ and also more gas refill compared to the $50(\mathrm{xx})^{*} 2$ pulse for the same value of $(\mathrm{xx})$. This would result in a higher argon density in the near cathode region and less self-sputtering. In combination with more opportunities for the ionized flux to escape the cathode, higher deposition rates are expected for the $25(\mathrm{xx}) * 4$ pulse compared to $50(\mathrm{xx}) * 2$, as seen in Fig. 3.

While a c-HiPIMS macropulse can theoretically consist of $5 \mu \mathrm{s}$ micropulses (as used by Konstantinidis et $a l .{ }^{14}$ ), in practice, the pulse power supply requirements to achieve peak powers of any reasonable magnitude in a HiPIMS process prevent the use of such micropulses with the present system, which uses a cathode ten times larger than that used by Konstantinidis et al.

\section{CONCLUSIONS}

It is clear that the use of chopped HiPIMS pulses has a significant influence on the deposition rate when sputtering titanium at similar voltages. Similar peak currents suggest similar high levels of ion-to-atom ratio for HiPIMS, while shorter pulse-on times may result in reduced gas heating with gas refill during the $t_{\mu \text { off }}$ periods. Further, changes to the $\mathrm{t}_{\mu \text { off }}$ times are shown to also impact deposition rate. At present, the results presented cannot be definitively explained, although relevant discussion has been presented to explain an increase of nearly $50 \%$ for c-HiPIMS with longer $\mathrm{t}_{\mu \text { off }}$ times when compared to HiPIMS. However, in order to more fully understand the mechanisms that are occurring with c-HiPIMS, calculations to assess the gas flow during cHiPIMS and investigations of the sputtered flux are on-going and these findings will be presented shortly.

\section{Nomenclature}

Macropulse = total "on" pulse sequence, with one per repetition frequency

Micropulse $=$ pulses that constitute the macropulse $t_{\text {on }}=$ total on-time of the entire macropulse

$\mathrm{t}_{\mu \mathrm{on}}=$ on-time of the micropulse

$\mathrm{t}_{\mu \text { off }}=$ off-time between the micropulses

$\mathrm{t}_{\mathrm{off}}=$ the time from the end of a macropulse until the start of the next

\section{ACKNOWLEDGMENT}

The authors gratefully acknowledge support from the Swiss State Secretariat for Education and Research in the framework of the COST Action PM0804.

${ }^{1}$ V. Kouznetsov, K. Macák, J. M. Schneider, U. Helmersson, and I. Petrov, Surf. Coat. Technol. 122, 290 (1999).

${ }^{2}$ K. Sarakinos, J. Alami, and S. Konstantinidis, Surf. Coat. Technol. 204, 1661 (2010).

${ }^{3}$ A. P. Ehiasarian, P. E. Hovsepian, L. Hultman, and U. Helmersson, Thin Solid Films 457, 270 (2004).

${ }^{4}$ M. Samuelsson, D. Lundin, J. Jensen, M. A. Raadu, J. T. Gudmundsson, and U. Helmersson, Surf. Coat. Technol. 205, 591 (2010).

${ }^{5}$ E. Wallin and U. Helmersson, Thin Solid Films 516, 6398 (2008).

${ }^{6}$ G. West, P. Kelly, P. Barker, A. Mishra, and J. Bradley, Plasma Process. Polym. 6, S543 (2009).

${ }^{7}$ D. Lundin and K. Sarakinos, J. Mater. Res. 27, 780 (2012).

${ }^{8}$ U. Helmersson, M. Lattemann, J. Böhlmark, A. P. Ehiasarian, and J. T. Gudmundsson, Thin Solid Films 513, 1 (2006).

${ }^{9}$ J. T. Gudmundsson, Vacuum 84, 1360 (2010).

${ }^{10}$ V. Stranak, S. Drache, R. Bogdanowicz, H. Wulff, A.-P. Herrendorf, Z. Hubicka, M. Cada, M. Tichy, and R. Hippler, Surf. Coat. Technol. 206, 2801 (2012).

${ }^{11}$ J. Lin, J. J. Moore, W. D. Sproul, B. Mishra, J. A. Rees, Z. Wu, R. Chistyakov, and B. Abraham, Surf. Coat. Technol. 203, 3676 (2009).

${ }^{12}$ V. Stranak, A. P. Herrendorf, S. Drache, M. Cada, Z. Hubicka, M. Tichy, and R. Hippler, Appl. Phys. Lett. 100, 141604 (2012).

${ }^{13}$ A. Anders, Surf. Coat. Technol. 205, S1 (2011).

${ }^{14}$ S. Konstantinidis, J. P. Dauchot, M. Ganciu, A. Ricard, and M. Hecq, J. Appl. Phys. 99, 013307 (2006).

${ }^{15}$ S. Kadlec, Plasma Process. Polym. 4, S419 (2007).

${ }^{16}$ D. Horwat and A. Anders, J. Appl. Phys. 108, 123306 (2010).

${ }^{17}$ D. Lundin, N. Brenning, D. Jädernäs, P. Larsson, E. Wallin, M. Lattemann, A. Raadu, and U. Helmersson, Plasma Sources Sci. Technol. 18, 045008 (2009).

${ }^{18}$ T. Kubart, M. Aiempanakit, J. Andersson, T. Nyberg, S. Berg, U. Helmersson, Surf. Coat. Technol. 205, S303 (2011).

${ }^{19}$ N. Brenning, C. Huo, D. Lundin, M. A. Raadu, C. Vitelaru, G. D. Stancu, T. Minea, and U. Helmersson, Plasma Sources Sci. Technol. 21, 025005 (2012). 